\title{
El profesor de bachillerato: concepciones, retos y logros
}

Marthelena Guerrero Rodríguez, Gustavo Ornelas Rodríguez y María Antonieta Anabel Valencia García

\section{Datos sobre el nivel medio superior en el estado de Aguascalientes}

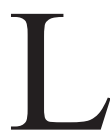

os datos estatales (IEA, 2016) señalan que en el ciclo escolar 2010-2011 se inscribieron en el nivel 46505 alumnos, mientras que en el ciclo escolar 2015-2016 la cifra es de 60451 (29 879 hombres

y 30572 mujeres), cabe hacer notar que sólo en el municipio de Aguascalientes cursan este nivel 44078 chicos, lo que habla de un aumento considerable en la matrícula; estos alumnos están inscritos en 310 planteles y son atendidos por una planta de 3350 docentes. Obsérvese el porcentaje de matrícula por tipo de sostenimiento (ver Gráfico 1).

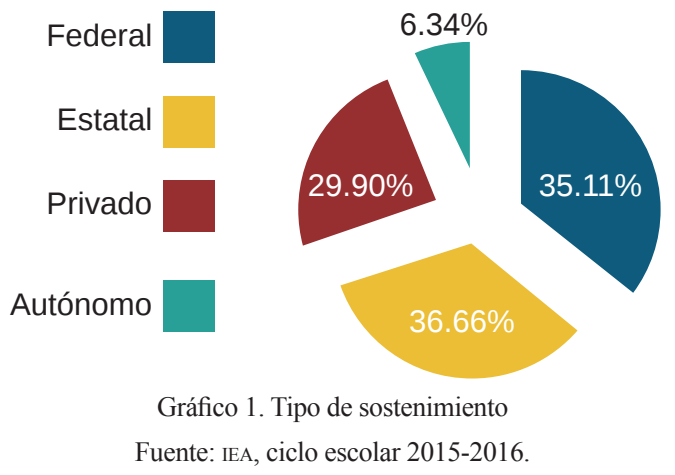

La cobertura a nivel estatal ha pasado de $65.15 \%$ en el ciclo escolar 2010-2011, a 79.98\% en el ciclo escolar 2015-2016. Aunque no hay datos claros sobre deserción, se sabe que es muy alta. El único dato que se encuentra está disgregado por el subsistema y va de $4.67 \%$ (BACHUAA) a $30.94 \%$ (DGECyTM). Lo mismo pasa con la reprobación, que va de $2.58 \%$ (DGECYTM) a $28.90 \%$ (DGeTI). (IEA, 2016; UAA 2017a; UAA 2017b; UAA 2017c).

Una de las principales dificultades originadas por la gran cantidad de opciones de formación académica en este nivel, era la poca posibilidad de movilidad de estudiantes entre un programa de estudio a otro diferente, lo que truncaba los estudios de los jóvenes, viéndose obligados a abandonar la escuela, o bien renunciar a lo ya cursado e iniciar a partir del primer semestre en el nuevo programa.
Actualmente, se ha logrado la portabilidad de estudios, la cual consiste en el reconocimiento de las unidades de aprendizaje curricular acreditadas, sin importar el grado o subsistema de educación media superior en que el alumno lo haya cursado (SEP, 2010: 17).

Con la instrumentación de la Reforma Integral de la Educación Media Superior (RIEMS) en el 2008, el sistema educativo de bachillerato a nivel nacional comienza una transformación, teniendo como principales objetivos mejorar la calidad, equidad, cobertura y pertinencia. Su propósito es la construcción de un Sistema Nacional de Bachillerato (SNB) en un marco de diversidad, en donde se integran y articulan las diferentes opciones de formación a partir de la definición de competencias genéricas, disciplinares y profesionales, definiendo así el Marco Curricular Común. En este sentido, la RIEMS se despliega y desarrolla a través de cuatro ejes generales: la construcción de un Marco Curricular Común (MCC) basado en competencias; la definición y regulación de la oferta educativa (modalidades); la instrumentación de mecanismos de gestión que permitan el adecuado tránsito de la propuesta; y un esquema de certificación para los egresados del SNB (SEP, 2010).

Otra situación a considerar es la falta de preparación pedagógica de la plantilla docente, 
lo anterior debido a que los profesores que atienden a los jóvenes bachilleres son egresados de distintas instituciones de educación superior con perfiles profesionales diversos, en su mayoría profesionistas que carecen de una preparación pedagógica en términos de su desempeño como docentes. Cabe señalar que la RIEMs tiene como fin transitar de un modelo de enseñanza y de aprendizaje tradicional, a un modelo que fomente la adquisición de competencias, lo que significa que es necesario comenzar a desarrollar acciones que permitan no sólo atender las necesidades de adquisición de conocimientos de las asignaturas que imparten, sino que deberán desarrollar habilidades para proporcionar una educación integral a sus estudiantes.

Una situación más es que los programas están llenos de teoría y lejos de una cultura juvenil, que cada día experimenta nuevas cosas con avances tecnológicos vertiginosos, con problemáticas muy focalizadas y ni el plan de estudios ni la escuela le ayudan a resolver, lo que lleva a que muchos de estos estudiantes deserten de la institución. Conjuntamente con ello, al cambiar la pirámide educacional en nuestro país, en donde la educación básica se achica y el nivel siguiente tiene un crecimiento en su demanda, se requiere hacerlo más eficaz y eficiente para responder a las necesidades de los jóvenes y la sociedad.

El nuevo Modelo Educativo (NME), de manera general, plantea una posible solución, ya que en su primer eje, sobre el planteamiento curricular, señala fortalecer el MCC basado en competencias, realizar una mejor selección de contenidos para el desarrollo de aprendizajes clave y la incorporación formal de las habilidades socioemocionales al currículo; en su segundo eje, respecto a colocar la escuela al centro, promueve el desarrollo de ambientes adecuados y pertinentes para el aprendizaje y el impulso al trabajo colegiado docente; en su tercer eje, sobre formación y desarrollo profesional docente, instala el sistema de evaluación del servicio profesional docente, el impulso de la oferta diversificada de formación continua; en cuanto a su cuarto eje, sobre inclusión y equidad, promueve los principios de equidad en el acceso y permanencia de los estudiantes que se encuentran en una situación de riesgo, rezago o desventaja; por último, en su quinto eje, sobre la gobernanza del
《 Con la instrumentación de la Reforma Integral de la

Educación Media Superior

(RIEMS), comienza una

transformación, para mejorar la

calidad, equidad, cobertura y

pertinencia, en un marco de

diversidad, en donde se

integran y articulan diferentes

opciones de formación, a partir

de la definición de

competencias genéricas,

disciplinares y profesionales,

en un Marco Curricular Común. $\gg$

sistema educativo, busca la creación de sistemas nacionales para el monitoreo y aseguramiento de la calidad educativa.

Como se puede observar, para la plantilla docente no sólo implica una mejor preparación académica, su obligada incorporación a un programa de actualización permanente y pertinente o la mediación efectiva del uso de tecnología y el trabajo colegiado, sino que además deberá ser sensible a las necesidades afectivas, sociales y de pertenencia que requieren los alumnos.

\section{El profesor de bachillerato: concepciones, retos y logros}

Ser profesor hoy en día implica estar en un mundo tecnologizado, hacer uso de las TIC como una herramienta es parte del reto cotidiano, porque atrás quedó el gis y el pizarrón, el libro y hasta las bibliotecas como las conocemos; junto a ello, debe pensar desde un mundo del conocimiento sin fronteras, en donde la transdisciplinariedad viene a dar respuesta al movimiento y la complejidad de esos conocimientos, en donde ser "especialista" es centrarse en una parte y perder el todo que rodea el saber de hoy, pues quedan fuera los planteamientos y las miradas mutilantes, reduccionistas y unidimensionales. Él debe tener claro que todo conocimiento es incompleto o inacabado; por lo tanto, no puede pensar en fenómenos complejos con métodos viejos. 


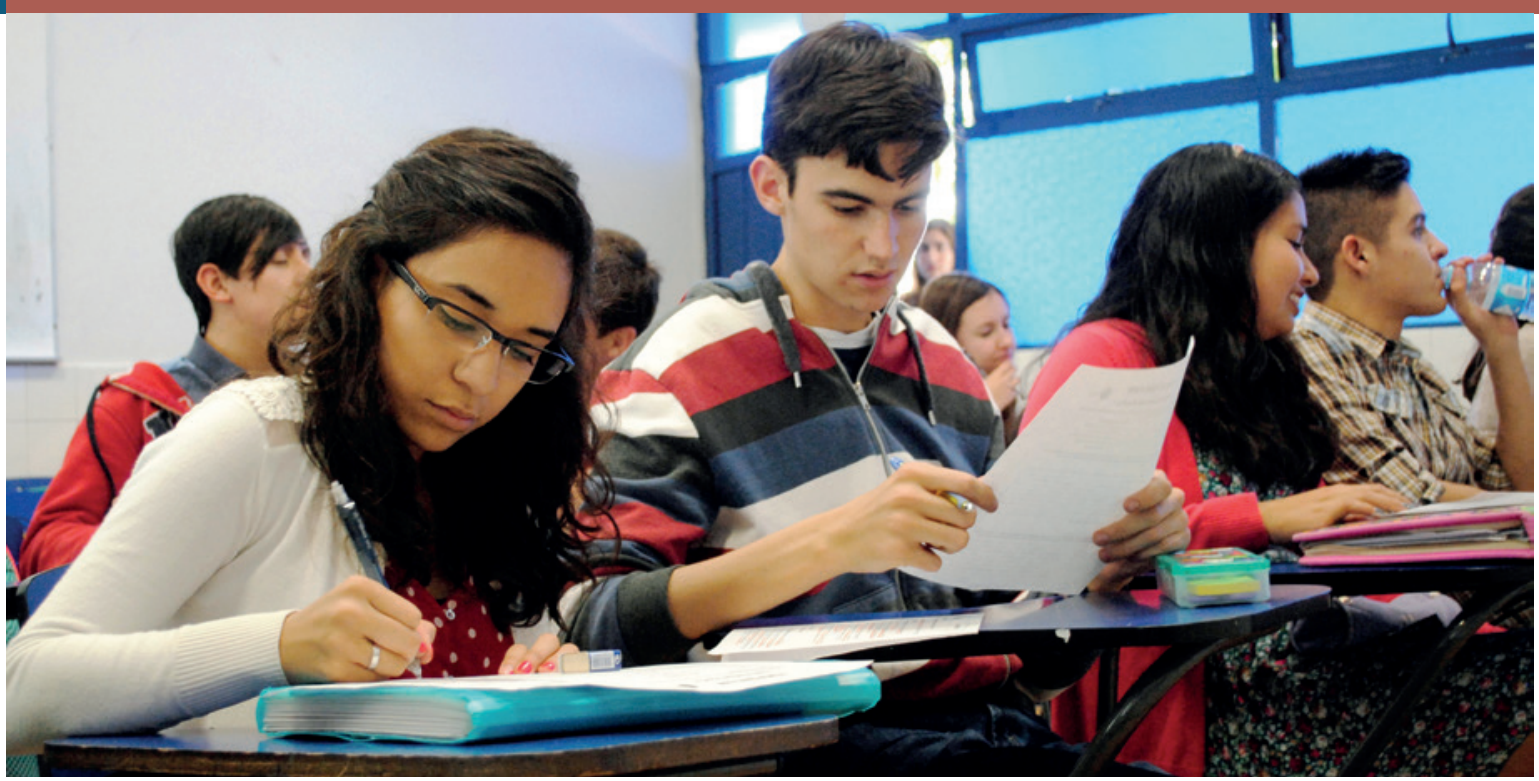

El profesor de hoy debe ser un acompañante activo en el trabajo que hace el joven para desarrollar sus diversas competencias, implica tener una visión del proceso que siguen y es construido por ellos; también, analizar la cotidianidad en que viven y conviven, para revisar y analizar cuáles son los problemas que le aquejan, de tal manera que lo que se planee y se realice en el aula esté situado en esa cotidianidad, en ese espacio cercano y vivido por los sujetos (Coll y Martín, 2006).

En este sentido, a casi ocho años de la implementación del SNB, debido a la complejidad del nivel, tanto el MCC como el modelo pedagógico que le subyace, aún están lejos de alcanzarse en los distintos planteles del país. La capacitación y actualización de los docentes del nivel debe ser un asunto prioritario para las autoridades educativas, pues son ellas precisamente quienes recrean el modelo por competencias.

La Universidad Pedagógica Nacional inicia en 21 de sus unidades la Maestría en Educación Media Superior (MEMS), como una respuesta a esa necesidad urgente de formar cuadros de profesores del nivel que respondan a los cambios que la sociedad exige y que, a su vez, contribuyan a la formación de los jóvenes, apoyando el desarrollo de sus competencias para la vida, lo que les permitirá, ya sea continuar estudios superiores, o bien insertarse en el mercado laboral.

Esta reflexión pretende dar cuenta de las concepciones que el profesor tiene de las competencias y cómo desarrollarlas en el estudiante de bachillerato, tomando como referencia a profesores del nivel medio superior, en específico 35 estudiantes de este posgrado que respondieron los siguientes cuestionamientos: ¿Qué son las competencias? ¿Qué implicaciones tiene para su práctica educativa trabajar bajo un modelo basado en competencias? ¿Qué dificultades ha tenido al implementar dicho modelo en su práctica docente? ¿Qué bondades le encuentra al trabajo bajo un modelo basado en competencias? (Tobón, 2008).

Sobre el concepto de "competencias" existe diversidad en las respuestas al concebirse como un "conjunto de conocimientos, habilidades, destrezas, capacidades y actitudes en la vida cotidiana", otros las definen en términos de "actividades que se realizan para demostrar el dominio en una función, trabajo o actividad específica". Algunos sólo las ubican como "habilidades que desarrolla un ser humano que le van a permitir desenvolverse en todos los ámbitos". Finalmente, las describen como "criterios de desempeño, integración de los saberes y capacidad de resolver problemas".

Ante el segundo cuestionamiento, mencionan que "implica aplicar métodos de enseñanza o actividades que involucren al estudiante a partir de problemas", ante ello se requiere "contar con instrumentos pertinentes para evaluar las competencias". La aplicación de este modelo "permite tener claridad del perfil de egreso, formar jóvenes capaces de poder crear, transformar y relacionar lo aprendido buscando una mejora personal y social". Reconocen que se da un "acompañamiento más eficaz en el proceso de enseñanza y aprendizaje" y reflexionan sobre la relevancia de que el docente debe desarrollarlas primero; 
sin embargo, identifican que existe una "falta de seguimiento hacia su práctica docente".

Sobre las dificultades que expresan en el tercer cuestionamiento, mencionan que un reto clave es "identificar el logro de las competencias o establecer un punto de partida para desarrollarlas; los grupos con los que se trabajan son numerosos, falta infraestructura, material, equipo y herramientas" para poder aplicar lo sugerido en los programas de estudio. Existe aún un "abuso en el dictado y elaboración de resúmenes, se mezclan modelos educativos"; se complica romper con lo tradicional, en donde "la memorización tiene un peso mayor y el conocimiento es generado únicamente por el profesor. Los alumnos muestran una baja confianza en ellos mismos por temor a fallar", lo que hace más lento el inicio o punto de partida en las actividades relacionadas con el uso de competencias y, por ende, "los contenidos de clase no son cubiertos en su totalidad. Los docentes no entendemos que una competencia no se logra con un solo docente y en una sola asignatura, el trabajo sigue dándose de forma desarticulada". Otra dificultad que expresan es el "uso de ejemplos prácticos en el aula, pues son ambientes con los que los alumnos no están familiarizados".

Finalmente, reconocen algunas bondades del modelo basado en competencias. Mencionaron que "se aprende a partir de problemas reales, por lo que el aprendizaje es más significativo". Los productos que se solicitan son más claros a partir de la especificidad de los indicadores de logro. La evaluación se realiza con base en actividades específicas, "existe mayor autonomía en el proceso de aprendizaje y mayor convivencia entre los estudiantes". Se conoce mejor al estudiante, ya que se identifican las potencialidades de los alumnos a partir de las evaluaciones continuas, los estudiantes son conscientes de su aprendizaje al aplicarlo en la vida cotidiana. En cuanto a los contenidos, se facilita una integración de los mismos al abordarse desde una realidad compleja. Para terminar, el enfoque basado en la productividad laboral "permite una inserción al mundo laboral de los egresados del nivel medio superior con mayor facilidad".

A partir de lo anterior, se identifican algunos retos en la formación de profesores del nivel medio superior, así como algunos logros que se han tenido al implementar un modelo basado en competencias a partir de la RIEMS. Se propone a los investigadores educativos centrarse en estos procesos formativos, para reconocer las áreas de oportunidad que existen y generar propuestas que atiendan estos nichos que finalmente impacten en el aprendizaje de los estudiantes y en la formación de ciudadanos que atiendan los retos complejos de la vida cotidiana, ante lo anterior

$[\ldots]$ es imprescindible un enfoque integrador [...] en el que la investigación y la ciencia formen parte de los currículos y de las preocupaciones de los profesores, en el que se reconozca la riqueza de la diversidad cultural y lingüística y en la apuesta por la innovación de las escuelas y de los profesores, sobre todo en redes y equipos de trabajo (OEI, 2008: 98).

\section{Fuentes de consulta}

Coll, C. y Martín, E. (2006). Vigencia del debate curricular. Aprendizajes básicos, competencias y estándares. México: SEP, Cuadernos de la Reforma SEP.

IEA (2016). Las cifras de la educación. Recuperado el 2 de junio de 2017, en: www.iea.gob.mx/ webiea/sistema_informacion/cifras_1415. aspx.

OEI (2008). Metas educativas 2021. La educación que queremos para la generación de los bicentenarios. España: OEI.

SEP (2010). Reforma Integral de la Educación Media Superior. Recuperado en junio de 2017, en: http://www.sep.gob.mx/work/models/ sep1/Resource/722/1/images/normmedsup1011.pdf.

Tobón, S. (2008). Diseño de módulos, didáctica y evaluación de competencias. Bogotá: Instituto CIFE.

UAA (2017b). Bachillerato Internacional del Centro de Educación Media. [Sitio web]. Recuperado el 15 de junio de 2017, en: www.uaa. $\mathrm{mx} /$ centros/cem/bi.php.

UAA (2017c). Bachillerato Oriente del Centro de Educación Media. [Sitio web]. Recuperado el 23 de junio de 2017, en: http://bit. ly/2h0O28n.

UAA (2017a). Universidad Autónoma de Aguascalientes. [Sitio web]. Recuperado el 9 de junio de 2017, en: www.uaa.mx. 